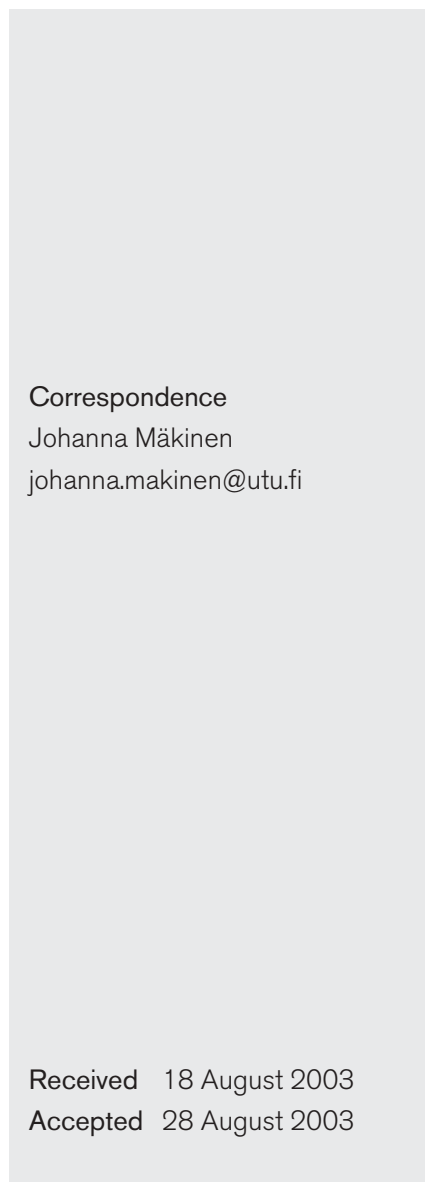

\title{
PFGE and pertactin gene sequencing suggest limited genetic variability within the Finnish Bordetella parapertussis population
}

\author{
Johanna Mäkinen, ${ }^{1,2}$ Jussi Mertsola, ${ }^{3}$ Hanna Soini, ${ }^{1}$ Heikki Arvilommi, ${ }^{1}$ \\ Matti K. Viljanen, ${ }^{4}$ Nicole Guiso ${ }^{5}$ and Qiushui $\mathrm{He}^{1}$ \\ 1,2National Public Health Institute, Department of Human Microbial Ecology and Inflammation ${ }^{1}$ and \\ Turku Graduate School of Biomedical Sciences, University of Turku², Turku, Finland \\ ${ }^{3}$ Department of Pediatrics, Turku University Hospital, Turku, Finland \\ ${ }^{4}$ Department of Medical Microbiology, University of Turku, Turku, Finland \\ ${ }^{5}$ Unité des Bordetella, Centre National de Référence des Bordetelles, Institut Pasteur, Paris, France
}

\begin{abstract}
The outer-membrane protein pertactin (Prn) of Bordetella pertussis, Bordetella parapertussis and Bordetella bronchiseptica is believed to function as an adhesin and is an important immunogen. The emergence of $B$. pertussis and $B$. bronchiseptica Prn variants has been reported. The aim of this study was to determine whether similar variation is found in $B$. parapertussis $\operatorname{Prn}$ and to characterize Finnish clinical B. parapertussis isolates that were collected in 1982-2000. Of 76 B. parapertussis isolates studied, seven ( $9 \%)$ were found to have silent and non-silent nucleotide changes. In addition, one (1\%) had eight POP repeats instead of nine. Three closely related $B$. parapertussis $X$ bal PFGE patterns were found. Genetic variation of $B$. parapertussis was found to be very limited, suggesting that $B$. parapertussis is a stable organism that is well-adapted to its own ecological niche.
\end{abstract}

\section{INTRODUCTION}

At the time of writing, the genus Bordetella includes eight species. Of these, Bordetella pertussis, Bordetella parapertussis and Bordetella bronchiseptica are closely related with relatively little genetic variation (Musser et al., 1986). The causative agent of whooping cough (pertussis), B. pertussis, is exclusively a human pathogen, whereas $B$. parapertussis and $B$. bronchiseptica may infect both humans and animals. In humans, $B$. parapertussis causes pertussis-like disease that is often milder than pertussis, although severe cases have been reported (Heininger et al., 1994; He et al., 1998). Much less is known about the epidemiology of $B$. parapertussis than about that of $B$. pertussis. When enhanced pertussis surveillance was carried out in Finland in 1994-1997 by using PCR and culture, about one-third of Bordetella cases were found to be caused by $B$. parapertussis, suggesting that the incidence of parapertussis is underestimated (He et al., 1998).

B. pertussis, B. parapertussis and B. bronchiseptica share a number of virulence factors. The most important difference

Abbreviation: Prn, pertactin.

The GenBank/EMBL/DDBJ accession numbers for the sequences of polymorphic regions 1 and 2 of Bordetella parapertussis pertactin are AF503929 and AF503928, respectively. between them is that pertussis toxin is produced only by B. pertussis; B. parapertussis and B. bronchiseptica possess, but do not express, the complete toxin operon. Pertactin (Prn), an outer-membrane protein that is expressed by B. pertussis, $B$. parapertussis and B. bronchiseptica, is an important virulence factor and is known to confer protective immunity to Bordetella infection in animals and humans (Charles et al., 1989; Kobisch \& Novotny, 1990; Leininger et al., 1992). Thus, it is a component of some acellular pertussis vaccines. Prn is proposed to function as an adhesin by promoting the attachment of bacteria to certain host cells via the RGD motif (Leininger et al., 1991). Prn has two regions that are composed of amino acid repeats (Charles et al., 1991): region 1 includes repeats of GGxxP (Charles et al., 1988), is located near the RGD motif and is polymorphic in B. pertussis (Mooi et al., 1998) and B. bronchiseptica (Boursaux-Eude \& Guiso, 2000; Register, 2001). Region 2 is composed of PQP repeats (Charles et al., 1988) and is polymorphic in B. bronchiseptica (Li et al., 1992; Boursaux-Eude \& Guiso, 2000; Register, 2001). Prns expressed by B. pertussis, B. parapertussis and B. bronchiseptica are highly similar $(>90 \%)$, with the major differences occurring in the number of repeats in regions 1 and 2 (Charles et al., 1988; Li et al., 1991, 1992). Polymorphism of B. pertussis and B. bronchiseptica Prn has been characterized, but variation of $B$. parapertussis Prn has not 
been observed previously (Boursaux-Eude \& Guiso, 2000). The aim of this study was to characterize the Prn proteins expressed by Finnish $B$. parapertussis isolates by sequencing part of the prn gene and to follow the recent evolution of the B. parapertussis population in Finland by using PFGE.

\section{METHODS}

Clinical isolates. In this study, we sequenced $76 \mathrm{~B}$. parapertussis isolates that were collected from patients resident in Finland during the years 1982-2000 (Table 1). Calcium alginate swabs and Regan-Lowe medium that contained charcoal agar and defibrinated sheep blood, supplemented with cephalexin, were used for primary cultures. After collection, swabs were inoculated onto plates at the local health centre or school. Plates were incubated in a humid atmosphere at $35^{\circ} \mathrm{C}$ and monitored daily for 7 days. Suspected colonies were Gram-stained and tested by slide agglutination with antisera to $B$. pertussis and B. parapertussis (Murex Biotech). The identity of the isolates was further confirmed by GLC and they were stored at $-70{ }^{\circ} \mathrm{C}$. Strains were collected from 12 different communities in south-western Finland (Sirkkala school and 11 geographically distinct communities). From two communities, strains were obtained consequentially with 5-6-year intervals. In six communities, there were outbreaks of B. parapertussis, which comprised more than three culture-confirmed cases that were considered to be epidemiologically related.

PCR, sequencing and PFGE. PCR, sequencing and PFGE were performed according to standardized recommendations for typing of B. pertussis (Mooi et al., 2000). Bacteria were cultivated on Regan-Lowe medium that contained charcoal agar and defibrinated sheep blood at $35^{\circ} \mathrm{C}$ for 2 days. For PFGE, B. pertussis strains 134 (USA), 287 (France) and B 902 (Sweden), French B. parapertussis strain CIP $64.11^{\mathrm{T}}$ and B. parapertussis ATCC $15311^{\mathrm{T}}$ were used as reference strains. Briefly, a $1000 \mathrm{bp}$ segment of the prn gene (covering regions 1 and 2) was sequenced from the $76 \mathrm{~B}$. parapertussis strains. Forty-seven of the strains, including the strains that possessed a variant form of Prn and at

Table 1. B. parapertussis clinical isolates used in this study

\begin{tabular}{|lrcc|}
\hline Community & $\begin{array}{c}\text { No. } \\
\text { isolates }\end{array}$ & $\begin{array}{c}\text { Year of } \\
\text { isolation }\end{array}$ & $\begin{array}{c}\text { No. Prn variants } \\
\text { found }\end{array}$ \\
\hline Sirkkala school & 16 & $1982-1983$ & 1 \\
Uittamo & 1 & 1990 & 0 \\
Paimio & 1 & 1993 & 0 \\
Salo & 2 & 1994 & 0 \\
Somero & 16 & 1994 & 3 \\
Littoinen & 14 & 1995 & 0 \\
Halikko & 1 & 1995 & 0 \\
Ulvila & 1 & 1996 & 1 \\
City of Turku & 1 & 1996 & 0 \\
Masku & 5 & 1996 & 1 \\
City of Tampere & 1 & 1996 & 0 \\
Salo & 2 & 1999 & 0 \\
Raisio & 12 & 2000 & $1^{*}$ \\
Somero & 3 & 2000 & 0 \\
Total & 76 & - & 7 \\
\hline
\end{tabular}

*This isolate had both the non-silent nucleotide change and eight PQP repeats instead of nine, arbitrarily designated as BPP prn3. least one invariant strain from each community, were genotyped by PFGE with the restriction enzymes SpeI and XbaI (New England Biolabs), with a CHEF Mapper II apparatus (Bio-Rad). PFGE reference strains and $\lambda$ ladder PFGE markers (New England Biolabs), used as molecular size standards, were included in each run. Nucleotide sequences were analysed with the Vector NTI Suite 6.0 software (InforMax) and compared by using the Vector NTI AlignX program with the CLUSTAL $\mathrm{W}$ algorithm. PFGE patterns were analysed both visually and with the assistance of Bionumerics version 2.5 software (Applied Maths) by using UPGMA with the Dice coefficient and $1 \%$ position tolerance settings for cluster analysis.

Western blotting. Prn expression was demonstrated by Western blotting using an anti-Prn mAb, BPE3 (Brennan et al., 1988) (data not shown). Bacterial cell lysates $\left(20 \times 10^{6}\right.$ bacteria per well $)$ and purified Prn protein (144 ng per well), provided by GlaxoSmithKline, were separated by SDS-PAGE on a $10 \%$ resolving gel. Proteins were transferred to a Hybond ECL nitrocellulose membrane (Amersham Pharmacia Biotech), which was blocked with $5 \%$ milk powder $/ 0 \cdot 1 \%$ Tween 20/PBS at $4{ }^{\circ} \mathrm{C}$ overnight. After washing, the membrane was incubated with a $1: 1000$ dilution of BPE3 antibody (obtained from Michael Brennan, FDA, Bethesda, USA) at $25^{\circ} \mathrm{C}$ for $1 \mathrm{~h}$. Immunochemical detection was performed with $1: 1000$-diluted horseradish peroxidase-conjugated rabbit anti-mouse immunoglobulins (DAKO A/ S), using an enhanced chemiluminescence system (Amersham), and analysed visually as positive or negative.

\section{RESULTS AND DISCUSSION}

Of the 76 B. parapertussis isolates studied, seven (9\%) were found to have both silent and non-silent nucleotide changes (arbitrarily designated as BPP Prn2). In addition, one (1\%) had eight PQP repeats instead of nine (arbitrarily designated as BPP Prn3) (Table 1 and Fig. 1). The silent mutation occurred at nucleotide $1078(\mathrm{C} \rightarrow \mathrm{T})$. The non-silent mutations change the codon of glutamine (Q311) to serine (S). S and $Q$ are both polar amino acids with uncharged side chains that are usually found at the surface of water-soluble proteins, where they contribute to both the water solubility and formation of binding sites for charged molecules. Therefore, the predicted $\mathrm{Q} \rightarrow \mathrm{S}$ change would probably not affect the conformation or function of Prn. However, to confirm this, the functionality of these Prn variants should be studied. In one of the variant isolates, the predicted amino acid sequence has eight $\mathrm{PQP}$ repeats instead of nine in region 2. The effect of variation in the number of PQP repeats on protein function is not known. Variation in region 2 has been observed in only two $B$. pertussis isolates, but in several isolates of B. bronchiseptica (Boursaux-Eude \& Guiso, 2000; Register, 2001). However, variation in region 2 of B. pertussis has only been investigated in a limited number of studies.

The presence of Prn in bacterial cell lysates was analysed by Western blotting using the anti-Prn mAb BPE3. It was confirmed that the variant forms of $B$. parapertussis Prn are expressed. However, the method was not quantitative; thus, we do not know whether the level of protein expression was the same in all isolates.

Three closely related $B$. parapertussis XbaI PFGE patterns were found among the 47 isolates studied. The isolates were considered to be closely related, as the PFGE patterns differed 


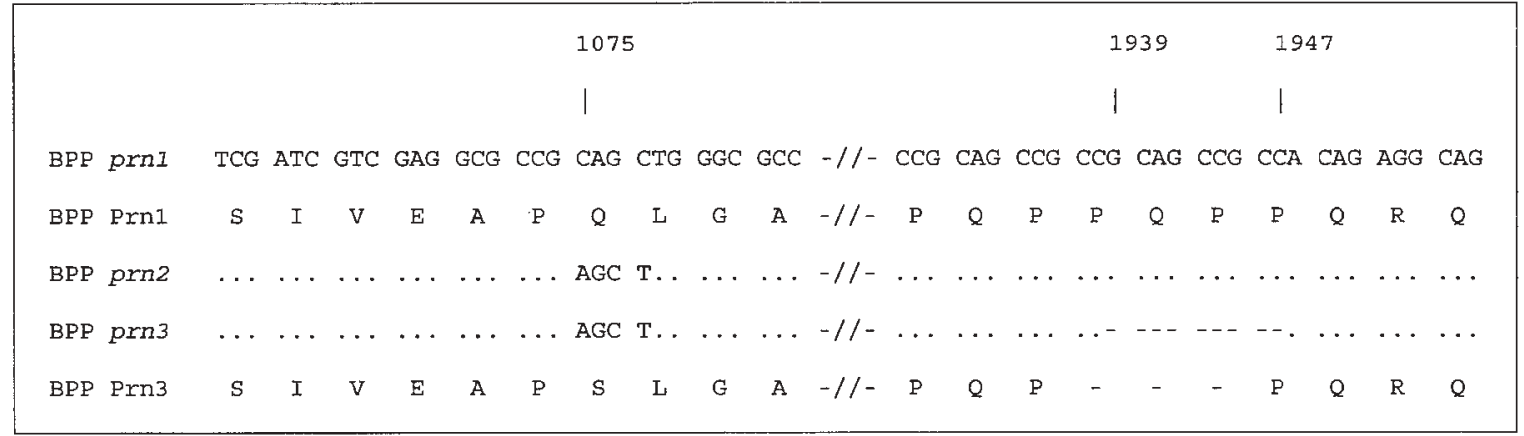

Fig. 1. Finnish B. parapertussis pertactin variants. DNA and corresponding amino acid sequences of polymorphic regions are depicted. Dots indicate identical bases; lines indicate deletion of bases. Numbers refer to position of bases in the pertactin gene. Complete sequences can be obtained from GenBank, accession numbers AF503929 (polymorphic region 1) and AF503928 (polymorphic region 2).

by only one or two bands (there was $96 \cdot 21 \%$ similarity between the profiles). These changes are consistent with a single genetic event, i.e. a point mutation or an insertion or deletion of DNA (Tenover et al., 1995). The isolates collected in 1982-1995 $(n=30)$ exhibit an A1 pattern (Fig. 2a). The majority of the isolates collected in $1996(n=6)$ exhibit an
A2 pattern, like reference strains ATCC $15311^{\mathrm{T}}$ and CIP $64.11^{\mathrm{T}}$, and only one isolate exhibited the A1 pattern. The A2 pattern is slightly different from the A1 pattern and is characterized by a fragment of $262 \mathrm{~kb}$ instead of the $250 \mathrm{~kb}$ fragment (Fig. 2a). In 1999-2000, all isolates $(n=15)$ exhibited an A2 pattern. The isolate that harbours eight
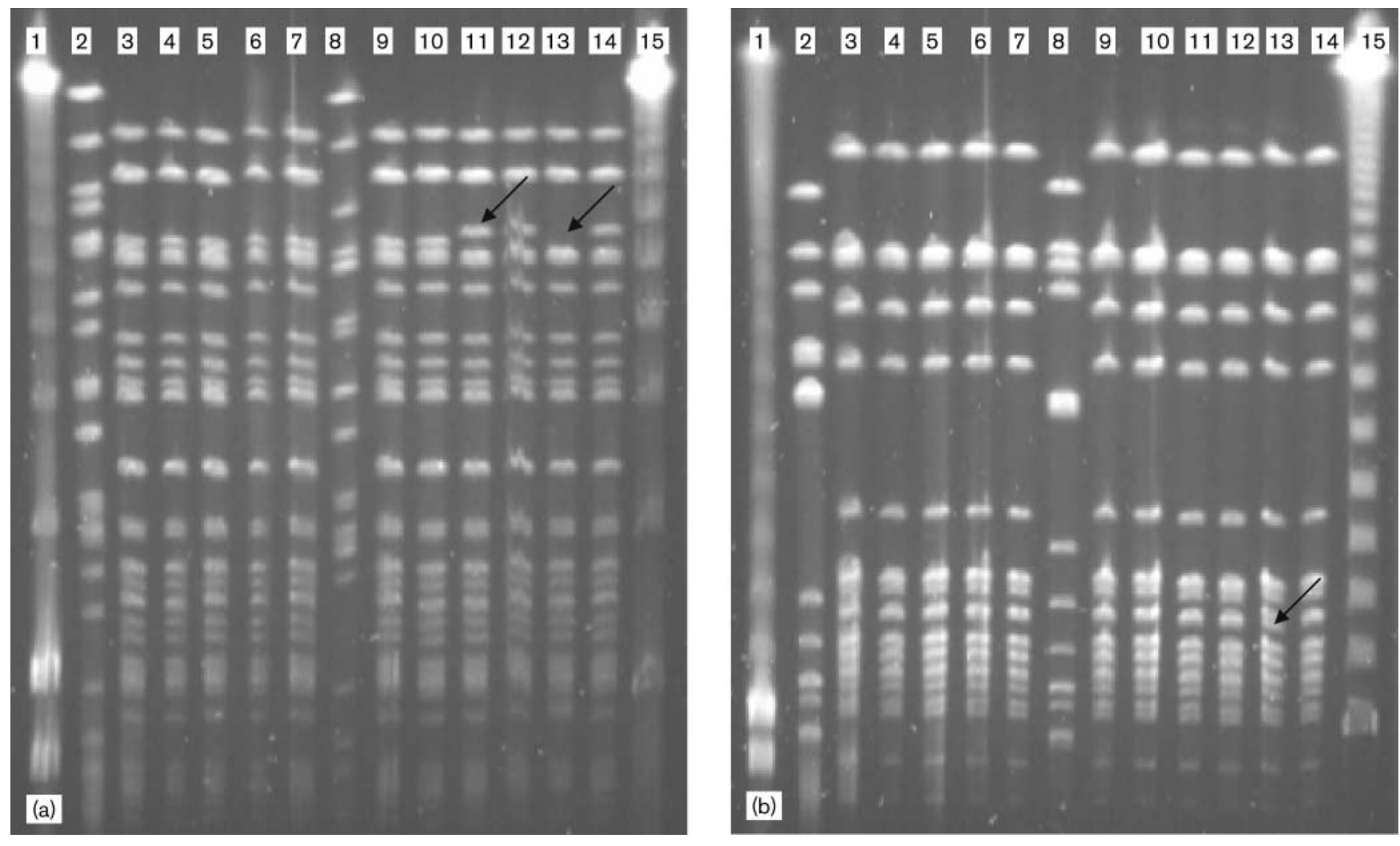

Fig. 2. PFGE profiles of (a) Xbal- and (b) Spel-digested chromosomal DNA of B. parapertussis isolates. Lanes 1 and 15, molecular

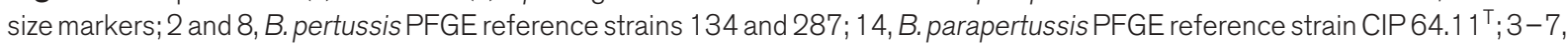
9, 10, DNA from isolates harbouring PFGE pattern A1 (isolated in 1982-1995); 11 and 12, DNA from isolates harbouring pattern A2 (isolated in 1999 and 2000); 13, DNA from the isolate harbouring pattern B (isolated in 2000). 
PQP repeats exhibits a unique PFGE type, arbitrarily designated as pattern B (Fig. 2a). Other isolates from the same outbreak that were submitted to PFGE $(n=6)$ exhibit the predominant pattern, A2. XbaI was found to be more discriminatory than SpeI, as patterns A1 and A2 could not be distinguished when examined with the restriction enzyme SpeI (Fig. 2b). The isolate that harbours the XbaI B pattern also produced a unique SpeI restriction pattern. As we only observed one B-type isolate, which contained eight PQP repeats, it remains to be seen whether the B-type strains will emerge in Finland and whether this PFGE pattern correlates with Prn type. However, there is no correlation between PFGE types A1 and A2 and prn sequences.

We do not know the importance of differences between groups A1 and A2 on the pathogenicity of B. parapertussis isolates. However, the same XbaI PFGE patterns were observed during a vaccine trial in 1992 and 1993 in Italy (Mastrantonio et al., 1998); the PFGE patterns of the isolates did not correlate with duration of coughing or severity of illness. In Italy, both strain types were co-circulating at the time, but the A1 isolates were found to be limited to the northern regions of the country (Mastrantonio et al., 1998). In Finland, it seems that type A2 has recently replaced type A1. However, to confirm this, historical Finnish B. parapertussis isolates would be needed. Unfortunately, Finnish $B$. parapertussis isolates collected before 1982 are not available.

In conclusion, analysis of the $B$. parapertussis isolates obtained from various communities in Finland during the past 20 years shows that the $B$. parapertussis population is very homogeneous, confirming the results of previous studies (Yuk et al., 1998; Boursaux-Eude \& Guiso, 2000). In this study, we showed that B. parapertussis Prn of seven of 76 isolates $(9 \%)$ had both non-silent and silent mutations and that one of the seven isolates had eight PQP amino acid repeats instead of nine in the second domain that contains repeated sequences (a domain that is very polymorphic in $B$. bronchiseptica, but not in B. pertussis). Several studies indicate that natural selection favours residue charge changes in the surface proteins of pathogens (Hughes, 1999), possibly allowing the pathogen to escape host defences. This might not be the case with $B$. parapertussis Prn, where Q311 can be changed to a similar amino acid, S. Our data confirm that repeated regions of $B$. parapertussis are very important for protein function, as they are conserved. It has been suggested that B. pertussis Prn variants have emerged as a result of vaccine-driven evolution, as strains used for whole-cell vaccines harbour a different Prn allele from that of most of the currently circulating B. pertussis strains (Mooi et al., 1998). The low level of variation in B. parapertussis Prn could indicate that $B$. parapertussis is not under similar selection pressure and has therefore remained stable. However, PFGE analysis clearly shows that the $B$. parapertussis population that infects humans is highly clonal and, thus, differences seen in the level of antigenic variation probably reflect differences in the overall stability of these organisms, rather than different selection pressures. Currently, acellular vaccines that contain only a few $B$. pertussis antigens are replacing whole-cell vaccines in the industrialized world. Surveillance should be continued to address whether the vaccine change affects the incidence of infection by B. parapertussis.

\section{ACKNOWLEDGEMENTS}

We thank Tuula Rantasalo, Anna Musku and Birgitta Aittanen for technical assistance, Michael Brennan, FDA (Bethesda, USA) for providing mAbs and Elizabeth Njamkepo and Valerie Caro, Institut Pasteur, Paris, France, for teaching the PFGE technique. The Academy of Finland, the Special Governmental Fund for University Hospitals (EVO) and the European Commission Quality of Life Programme (QLK2-CT-2001-01819) supported this work financially.

\section{REFERENCES}

Boursaux-Eude, C. \& Guiso, N. (2000). Polymorphism of repeated regions of pertactin in Bordetella pertussis, Bordetella parapertussis, and Bordetella bronchiseptica. Infect Immun 68, 4815-4817.

Brennan, M. J., Li, Z. M., Cowell, J. L., Bisher, M. E., Steven, A. C., Novotny, P. \& Manclark, C. R. (1988). Identification of a 69-kilodalton nonfimbrial protein as an agglutinogen of Bordetella pertussis. Infect Immun 56, 3189-3195.

Charles, I. G., Dougan, G., Pickard, D., Charfield, S., Smith, M., Novotny, P. \& Fairweather, N. (1988). Molecular cloning and analysis of P. 69, a vir-controlled protein from Bordetella pertussis. Tokai J Exp Clin Med 13 (Suppl.), 227-234.

Charles, I. G., Dougan, G., Pickard, D., Chatfield, S., Smith, M., Novotny, P., Morrissey, P. \& Fairweather, N. F. (1989). Molecular cloning and characterization of protective outer membrane protein P.69 from Bordetella pertussis. Proc Natl Acad Sci U S A 86, 3554-3558.

Charles, I. G., Li, J. L., Roberts, M. \& 13 other authors (1991). Identification and characterization of a protective immunodominant B cell epitope of pertactin (P.69) from Bordetella pertussis. Eur J Immunol 21, 1147-1153.

He, Q., Viljanen, M. K., Arvilommi, H., Aittanen, B. \& Mertsola, J. (1998). Whooping cough caused by Bordetella pertussis and Bordetella parapertussis in an immunized population. JAMA 280, 635-637.

Heininger, U., Stehr, K., Schmitt-Grohe, S., Lorenz, C., Rost, R., Christenson, P. D., Uberall, M. \& Cherry, J. D. (1994). Clinical characteristics of illness caused by Bordetella parapertussis compared with illness caused by Bordetella pertussis. Pediatr Infect Dis J 13, 306-309.

Hughes, A. L. (1999). Adaptive Evolution of Genes and Genomes. New York: Oxford University Press.

Kobisch, M. \& Novotny, P. (1990). Identification of a 68-kilodalton outer membrane protein as the major protective antigen of Bordetella bronchiseptica by using specific-pathogen-free piglets. Infect Immun $\mathbf{5 8}$, 352-357.

Leininger, E., Roberts, M., Kenimer, J. G., Charles, I. G., Fairweather, N., Novotny, P. \& Brennan, M. J. (1991). Pertactin, an Arg-Gly-Aspcontaining Bordetella pertussis surface protein that promotes adherence of mammalian cells. Proc Natl Acad Sci U S A 88, 345-349.

Leininger, E., Ewanowich, C. A., Bhargava, A., Peppler, M. S., Kenimer, J. G. \& Brennan, M. J. (1992). Comparative roles of the Arg-Gly-Asp sequence present in the Bordetella pertussis adhesins pertactin and filamentous hemagglutinin. Infect Immun 60, 2380-2385.

Li, L. J., Dougan, G., Novotny, P. \& Charles, I. G. (1991). P.70 pertactin, an outer-membrane protein from Bordetella parapertussis: cloning, nucleotide sequence and surface expression in Escherichia coli. Mol Microbiol 5, 409-417.

Li, J., Fairweather, N. F., Novotny, P., Dougan, G. \& Charles, I. G. (1992). 
Cloning, nucleotide sequence and heterologous expression of the protective outer-membrane protein P.68 pertactin from Bordetella bronchiseptica. J Gen Microbiol 138, 1697-1705.

Mastrantonio, P., Stefanelli, P., Giuliano, M., Herrera Rojas, Y., Ciofi degli Atti, M., Anemona, A. \& Tozzi, A. E. (1998). Bordetella parapertussis infection in children: epidemiology, clinical symptoms, and molecular characteristics of isolates. J Clin Microbiol 36, 999-1002.

Mooi, F. R., van Oirschot, H., Heuvelman, K., van der Heide, H. G. J., Gaastra, W. \& Willems, R. J. L. (1998). Polymorphism in the Bordetella pertussis virulence factors P.69/pertactin and pertussis toxin in the Netherlands: temporal trends and evidence for vaccine-driven evolution. Infect Immun 66, 670-675.

Mooi, F. R., Hallander, H., Wirsing von König, C. H., Hoet, B. \& Guiso, N. (2000). Epidemiological typing of Bordetella pertussis isolates: recommendations for a standard methodology. Eur J Clin Microbiol Infect Dis 19, $174-181$.
Musser, J. M., Hewlett, E. L., Peppler, M. S. \& Selander, R. K. (1986). Genetic diversity and relationships in populations of Bordetella spp. J Bacteriol 166, 230-237.

Register, K. B. (2001). Novel genetic and phenotypic heterogeneity in Bordetella bronchiseptica pertactin. Infect Immun 69, 1917-1921.

Tenover, F. C., Arbeit, R. D., Goering, R. V., Mickelsen, P. A., Murray, B. E., Persing, D. H. \& Swaminathan, B. (1995). Interpreting chromosomal DNA restriction patterns produced by pulsed-field gel electrophoresis: criteria for bacterial strain typing. J Clin Microbiol 33, 2233-2239.

Yuk, M. H., Heininger, U., Martinez de Tejada, G. \& Miller, J. F. (1998). Human but not ovine isolates of Bordetella parapertussis are highly clonal as determined by PCR-based RAPD fingerprinting. Infection 26, 270-273. 